\section{How I treat relapsed and/or refractory multiple myeloma}

\author{
Hans C. Lee, ${ }^{1}$ Claudio Cerchione ${ }^{2}$ \\ ${ }^{1}$ The University of Texas MD Anderson \\ Cancer Center, Department of \\ Lymphoma/Myeloma, Houston, TX, \\ USA; ${ }^{2}$ Hematology Unit, Istituto \\ Scientifico Romagnolo per lo Studio e la \\ Cura dei Tumori (IRST) IRCCS, \\ Meldola (FC), Italy
}

\begin{abstract}
The expanding therapeutic landscape of relapsed and/or refractory multiple myeloma (RRMM) has contributed to significant improvements in patient outcomes. These have included combinations of proteasome inhibitors (PIs), immunomodulatory drugs (IMiDs), monoclonal antibodies (mAbs), histone deacetylase inhibitors, and/or alkylating agents. More recently, the approval of the first-in-class nuclear export inhibitor selinexor and the first-in-class B-cell maturation antigen (BCMA) antibody-drug conjugate (ADC) belantamab mafodotin has helped address the current unmet need in patients refractory to PI, IMiD, and antiCD38 mAb directed therapy, otherwise known as triple class refractory myeloma. With the growing number of treatment options in the RRMM therapeutic landscape, the choice and sequencing of drugs and combinations has become increasingly complex. In this review we discuss our approach and considerations in the treatment of both early and late RRMM based on best available data and our clinical experience.
\end{abstract}

\section{Introduction}

Outcomes in multiple myeloma patients have improved substantially over the last 10-15 years due to the incorporation of immunomodulatory drugs (IMiDs), proteasome inhibitors (PIs), and monoclonal antibodies (mAbs) to standard myeloma treatment regimens. In relapsed and/or refractory multiple myeloma (RRMM), a number of treatment options exist based on randomized phase 3 trials that have led to the regulatory approval of various combinations of PIs, IMiDs, mAbs targeting CD38 or SLAMF7, and histone deacetylase inhibitors. Moreover, based on recent phase 2 studies, the first-in-class nuclear export inhibitor selinexor and the first-in-class B- cell maturation antigen (BCMA) antibodydrug conjugate (ADC) belantamab mafodotin were recently approved, helping address an unmet need in myeloma refractory to PIs, IMiDs, and anti-CD38 mAbs, otherwise known as triple-class refractory myeloma. With the growing number of treatment options in the RRMM therapeutic landscape, the choice and optimal sequencing of agents has become increasingly complex. In this review we discuss our approach and considerations in the treatment of RRMM based on the best available data and our clinical experience through several representative cases. While the preferred approach is to enroll on a clinical trial, we will focus our discussion on drugs and regimens that are currently commercially available for use in routine clinical practice.

\section{Case 1}

A 64 year-old woman was diagnosed with IgG kappa multiple myeloma with lytic bone lesions and anemia (hemoglobin $8.8 \mathrm{~g} / \mathrm{dL}$ ) on initial presentation. Initial Mprotein was $3.6 \mathrm{~g} / \mathrm{dL}$. Fluorescence in situ hybridization (FISH) demonstrated standard risk disease with del 13q. She was treated with frontline therapy with bortezomib, lenalidomide, and dexamethasone for four cycles, followed by high-dose melphalan and autologous stem cell transplantation (ASCT). Subsequently she was started on maintenance lenalidomide, achieving a compete response (CR) to therapy. However, 34 months after her ASCT, she now has evidence of a new lytic lesion in her right humerus on positron emission tomography/computed tomography (PET/CT) and reappearance of her M-protein at $0.8 \mathrm{~g} / \mathrm{dL}$.

\section{Case 1: discussion}

The patient in Case 1 represents probably the most common scenario encountered at first relapse in myeloma today given the prevalence of maintenance lenalidomide use in both transplant and non-transplant patients. In this case, the patient has both biochemical progression and clinical relapse, warranting a change in therapy.

In a daratumumab naïve, lenalidomide refractory patient, incorporating anti-CD38 directed therapy in the patient's 2 nd line of therapy would be our treatment of choice. Several randomized trials in early RRMM have demonstrated the benefit of combining anti-CD38 mAbs and PIs, which would provide a class switch away from an IMiDbased regimen in this case. Daratumumab in combination with bortezomib and dexam-
Correspondence: Hans C. Lee, Department of Lymphoma/Myeloma, The University of Texas MD Anderson Cancer Center, 1515 Holcombe Blvd, Unit 429, Houston, TX, 77030, USA. Tel: 713-745-8430.

E-mail: hclee@mdanderson.org

Key words: Multiple myeloma; Relapsed; Refractory.

Acknowledgements: H.C.L. would like to acknowledge support from the Baer Family Fund and the Goff-Street Foundation.

Conflict of interest: H.C.L. declares consulting fees from Amgen, Celgene, GlaxoKlineSmith, Janssen, Sanofi, and Takeda and research funding from Amgen, Celgene, Daiichi Sankyo, GlaxoKlineSmith, Janssen, Regeneron, and Takeda.

This work is licensed under a Creative Commons Attribution-NonCommercial 4.0 International License (CC BY-NC 4.0).

(c) Copyright: the Author(s), 2020

Licensee PAGEPress, Italy

Hematology Reports 2020; 12(s1):8955

doi:10.4081/hr.2020.8955

ethasone (DVd) was the first anti-CD38 $\mathrm{mAb}$ and $\mathrm{PI}$ combination to gain regulatory approval based on the CASTOR study which showed an improvement in progression free survival (PFS) compared to bortezomib and dexamethasone (Vd). ${ }^{1}$ However, among $18 \%$ of patients in the DVd arm who were refractory to lenalidomide in their last line of therapy, median PFS was only 9.3 months. ${ }^{2}$ More recently, results from randomized phase 3 studies evaluating daratumumab (CANDOR) or isatuximab (IKEMA) in combination with the second generation PI carfilzomib and dexamethasone $(\mathrm{Kd})$ versus $\mathrm{Kd}$ alone have been reported. In the CANDOR study, among the subset of lenalidomide refractory patients, median PFS was significantly higher in the daratumumab-Kd arm (not-reached) versus the Kd arm (11.1 months, hazard ratio (HR) $0.47,95 \%$ confidence interval (CI) 0.29 0.78). ${ }^{3}$ Likewise among patients who were lenalidomide refractory in the IKEMA study, a beneficial trend was seen with the addition of isatuximab to $\mathrm{Kd}$ versus $\mathrm{Kd}$ alone (hazard ratio $0.60,95 \%$ confidence interval 0.34-1.06). ${ }^{4}$ When choosing an anti-CD38 $\mathrm{mAb}$ and PI combination, our preference would be daratumumab-Kd or isatuximab-Kd in this setting based on a stronger PFS efficacy signal compared to daratumumab-Vd. However, in older patients or those with pre-existing cardiac conditions, daratumumab-Vd should be 
considered. The use of the third generation IMiD in combination with an anti-CD38 $\mathrm{mAb}$ would also be an option in this setting. While randomized phase 3 data is awaited from the APOLLO study (NCT03180736) evaluating the benefit of adding daratumumab to pomalidomide and dexamethasone (Pd) in early RRMM, two phase 2 studies have demonstrated the strong efficacy of this combination. , $6^{-6}$ In particular, the phase 2 MM-014 study enrolled patients with early RRMM with 1-2 lines of prior therapy. Among 84 lenalidomide-refractory patients, median PFS was 21.8 months, suggesting that durable responses can be attained even without a class switch away from IMiD-based therapy in patients progressing on lenalidomide.

Based on these data, daratumumab-Pd is frequently utilized in our routine clinical practice in patients progressing on lenalidomide. Given several strong therapeutic options in this setting (daratumumab-Kd, isatuximab-Kd, and daratumumab-Pd), other important considerations include any patient comorbidities that may affect the tolerability of certain treatment options based on known drug adverse event profiles. In addition, patient preferences on route of administration (oral, subcutaneous, or intravenous) and frequency of clinic visits for treatment administration also becomes an important consideration.

\section{Case 2}

A 76 year-old man is diagnosed with kappa light chain myeloma with anemia (hemoglobin $8.3 \mathrm{~g} / \mathrm{dL}$ ) on presentation. Myeloma FISH studies demonstrated standard risk disease. He underwent induction therapy with bortezomib, lenalidomide, and dexamethasone for 8 cycles achieving a CR to therapy. Afterwards, due to personal preference, he stopped myeloma therapy and elected observation. Approximately 15 months later, he developed asymptomatic biochemical recurrence of disease that was initially observed but now has clear acceleration in the kinetics of disease progression with a serum free kappa light chain level of $330 \mathrm{mg} / \mathrm{L}$ and a free light chain ratio of ratio 44.2. A repeat bone marrow biopsy shows no high-risk FISH markers.

\section{Case 2: discussion}

Unlike case 1 , this patient is considered to have lenalidomide sensitive disease, despite previous exposure, given the prolonged period of time ( $>60$ days) between treatment discontinuation and disease progression. In this case, retreatment with a lenalidomide-based regimen would be a preferred choice. Options with regulatory approval based on randomized phase 3 data include elotuzumab in combination with lenalidomide and dexamethasone ( $\mathrm{Rd}$, ERd), ${ }^{7}$ ixazomib in combination with $\mathrm{Rd}$ (IRd). ${ }^{8}$ carfilzomib in combination with $\mathrm{Rd}$ $(\mathrm{KRd})^{9}$ and daratumumab in combination with Rd (DRd). ${ }^{10}$ Both ERd ${ }^{11}$ and $\mathrm{KRd}^{12}$ have demonstrated overall survival (OS) benefit with long term follow-up when compared to the Rd backbone alone, and it is likely that DRd will achieve similar results as data matures based on a median PFS of 45.8 versus 17.5 months in the Rd arm and strong HR ratio 0.43 (95\% CI 0.35 $0.54)$.

Given several options in this setting, therapeutic considerations may again depend on patient comorbidities that may affect the tolerability to certain drugs and patient preferences on route of administration. If efficacy was the only consideration, DRd would be our preferred option in this case given the fact that the patient is naïve to anti-CD38 mAb therapy and the impressive median PFS and median PFS2 of this combination that has been reported with longer follow-up. ${ }^{13}$

\section{Case 3}

A 55 year-old man was diagnosed with IgG kappa multiple myeloma with lytic bone lesions on presentation. FISH demonstrated $\mathrm{t}(11 ; 14)$ and amplification of $+1 \mathrm{q} 21$ with 4 copies of $C K S 1 B$. He was treated with bortezomib, lenalidomide, and dexamethasone for 3 cycles, followed by highdose melphalan and ASCT, followed by maintenance bortezomib, lenalidomide, and dexamethasone given his high-risk disease in a risk-adapted maintenance approach. His best response was a minimal residual disease (MRD) negative CR. After 29 months on maintenance therapy, patient had disease progression at which time he was treated with second line daratumumab-Pd. After 15 months on daratumumab-Pd, the patient now again has evidence of disease progression.

\section{Case 3: discussion}

This patient has had 2 lines of prior therapy and is now triple class refractory to PIs (bortezomib), IMiDs (lenalidomide, pomalidomide), and an anti-CD38 $\mathrm{mAb}$ (daratumumab). The patient is not refractory to the second generation PI carfilzomib and alkylating agents, and their use in combination with a regimen such as carfilzomib, cyclophosphamide, and dexametha- sone would be one option. ${ }^{14}$

The presence of $\mathrm{t}(11 ; 14)$ also makes the off-label use of the Bcl-2 inhibitor venetoclax a consideration. While the phase 3 BELLINI trial of venetoclax, bortezomib, and dexamethasone versus bortezomib and dexamethasone demonstrated a trend towards inferior OS in the venetoclax arm, a PFS benefit and a trend towards OS benefit was retained in the subset of patients with $t(11 ; 14) .{ }^{15}$ Preliminary safety and efficacy data have also been reported with carfilzomib, venetoclax, and dexamethasone with patients with $\mathrm{t}(11 ; 14)$ showing the strongest efficacy signal. ${ }^{16}$ The role of venetoclax is still evolving in RRMM as data continue to mature so should be used judiciously in this setting and be limited to $\mathrm{t}(11 ; 14)$ patients.

Other considerations of lower priority would be combining the histone deacetaylase inhibitor panobinostat with proteasome inhibitors. In particular, the combination of panobinostat, bortezomib, and dexamethasone is approved for RRMM patients who have received at least two prior regimens including bortezomib and an immunomodulatory agent (IMiD). While the pivotal PANORAMA 1 study that led to the regulatory approval of this regimen excluded bortezomib-refractory patients, ${ }^{17}$ the phase 2 PANORAMA 2 study enrolled only bortezomib-refractory patients which demonstrated an ORR $34.5 \%$, median duration of response 6 months, and median PFS of 5.4 months. ${ }^{18}$ Phase 1 and 2 data with the combination of carfilzomib and panobinostat have also been reported. ${ }^{15,19}$

While the patient has not been on an elotuzumab-IMiD combination, the expected NK depletion from recent daratumumab therapy may diminish any potential efficacy, ${ }^{20,21}$ given the role of NK cell-mediated antibody-dependent cellular cytotoxicity (ADCC) as a major mechanism of action of elotuzumab. Therefore, as the patient is also IMiD refractory, we would deprioritize the use of an elotuzumab-IMiD based combination in this setting.

\section{Case 4}

A 67 year-old woman presents to the clinic for discussion of treatment options for her relapsed IgA lambda multiple myeloma. She has had 6 lines of prior therapy including high-dose melphalan and autologous stem cell transplantation. She is refractory to lenalidomide, pomalidomide, bortezomib, carfilzomib, daratumumab, and cyclophosphamide. Most recently, she has been treated with isatuximab, pomalido- 
mide, and dexamethasone and now has progressive disease.

\section{Case 4: discussion}

This patient is both triple class refractory and penta-refractory to all five major drugs in myeloma treatment including lenalidomide, pomalidomide, bortezomib, carfilzomib and the anti-CD38 mAb daratumumab (and isatuximab). Hyperfractionated cyclophosphamide-based cytotoxic chemotherapy regimens such as DTPACE,${ }^{22}$ modified-CBAD, ${ }^{23}$ or DCEP, ${ }^{24}$ have historically been used in this setting but are often poorly tolerated in late RRMM and associated with high morbidity and mortality rates.

The recent regulatory approval of belantamab mafodotin (belamaf), the firstin-class BCMA antibody-drug conjugate (ADC) and from a broader standpoint, the first BCMA-targeted therapy, would be our preferred consideration in this patient. While the ORR of $31 \%$ at the approved 2.5 $\mathrm{mg} / \mathrm{kg}$ belamaf dose in the DREAMM-2 study is comparable to other recent singleagent approvals in RRMM, ${ }^{25-27}$ the depth of response ( $\geq$ VGPR 19\%) and median duration of response of 11 months were particularly promising. ${ }^{28}$ A multidisciplinary team of oncologists and eye care specialists is needed to safely treat patients with belamaf given its association with frequent yet reversible corneal ocular adverse events, which are managed effectively by dose delays and dose reductions based on ocular exam findings and symptoms.

The first-in-class oral nuclear export inhibitor selinexor would also be a consideration for this patient based on an ORR of $25 \%$, median DOR of 4.4 months, and median PFS of 4.7 months in the pivotal STORM registration study targeting triple class refractory myeloma patients. ${ }^{27}$ Aggressive supportive care is also important when administrating selinexor to mitigate adverse events, including prophylactic anti-nausea agents with a 5-HT3 antagonist (e.g. ondansetron) in combination with olanzapine and/or a neurokinin 1 (NK1) receptor antagonist. ${ }^{29}$

\section{Conclusions}

The therapeutic landscape in RRMM is rapidly evolving, in relation to both efficacy and treatment tolerability, which has led to continued improvement in the overall survival of myeloma patients over the last two decades. With a plethora of therapeutic options, particularly in early RRMM, the choice of therapy should also be individualized based on patient- and disease-related factors such as previous therapies, duration of prior responses, nature of relapse (biochemical or clinical), and patient comorbidities in relation to known drug adverse event profiles. In late RRMM, triple class refractory myeloma remains a therapeutic challenge, an area where the recent approvals of selinexor and belamaf have helped address. The anticipated approvals of other novel therapeutic agents such as BCMA-targeted chimeric antigen receptor T-cells (CAR-T) and bispecific antibody Tcell engagers will bolster this area of unmet need. While having many treatment options is clearly advantageous, the choice and sequencing of therapeutic options in RRMM remains a challenge in the absence of randomized clinical data that address common clinical scenarios.

\section{References}

1. Palumbo A, Chanan-Khan A, Weisel K, Nooka AK, Masszi T, Beksac M, et al. Daratumumab, Bortezomib, and Dexamethasone for Multiple Myeloma. N Engl J Med. 2016;375(8):754-66.

2. Spencer A, Lentzsch S, Weisel K, AvetLoiseau H, Mark TM, Spicka I, et al. Daratumumab plus bortezomib and dexamethasone. Haematologica. 2018;103(12):2079-87.

3. Usmani SZ, Quach H, Mateos M-V, Landgren O, Leleu X, Siegel DS, et al. Carfilzomib, Dexamethasone, and Daratumumab Versus Carfilzomib and Dexamethasone for the Treatment of Patients with Relapsed or Refractory Multiple Myeloma (RRMM): Primary Analysis Results from the Randomized, Open-Label, Phase 3 Study Candor (NCT03158688). Blood. 2019;134 (Supplement_2):LBA-6-LBA-.

4. Moreau P, Dimopoulos M, Mikhael J, Yong K, Capra M, Facon T, Hajek R, Spicka I, Risse ML, Asset G, Macé S, Martin T. Isatuximab Plus Carfilzomib and Dexamethasone vs Carfilzomib and Dexamethasone in Relapsed/Refractory Multiple Myeloma (IKEMA): Interim Analysis of A Phase 3 Randomized, Open-Label Study, LB2603. 25th Congress of the European Hematology Association. 2020.

5. Chari A, Suvannasankha A, Fay JW, Arnulf B, Kaufman JL, Ifthikharuddin JJ, et al. Daratumumab plus pomalidomide and dexamethasone in relapsed and/or refractory multiple myeloma. Blood. 2017;130(8):974-81.

6. Siegel DS, Schiller GJ, Samaras C, Sebag M, Berdeja J, Ganguly S, et al.
Pomalidomide, dexamethasone, and daratumumab in relapsed refractory multiple myeloma after lenalidomide treatment. Leukemia. 2020.

7. Lonial S, Dimopoulos M, Palumbo A, White D, Grosicki S, Spicka I, et al. Elotuzumab Therapy for Relapsed or Refractory Multiple Myeloma. N Engl J Med. 2015;373(7):621-31.

8. Moreau P, Masszi T, Grzasko N, Bahlis NJ, Hansson M, Pour L, et al. Oral Ixazomib, Lenalidomide, and Dexamethasone for Multiple Myeloma. N Engl J Med. 2016;374(17):1621-34.

9. Stewart AK, Rajkumar SV, Dimopoulos MA, Masszi T, Špička I, Oriol A, et al. Carfilzomib, lenalidomide, and dexamethasone for relapsed multiple myeloma. N Engl J Med. 2015;372(2):142-52.

10. Dimopoulos MA, Oriol A, Nahi H, SanMiguel J, Bahlis NJ, Usmani SZ, et al. Daratumumab, Lenalidomide, and Dexamethasone for Multiple Myeloma. N Engl J Med. 2016;375(14):1319-31.

11. Dimopoulos MA, Lonial S, White D, Moreau P, Weisel K, San-Miguel J, et al. Elotuzumab, lenalidomide, and dexamethasone in RRMM: final overall survival results from the phase 3 randomized ELOQUENT-2 study. Blood Cancer J. 2020;10(9):91.

12. Siegel DS, Dimopoulos MA, Ludwig $\mathrm{H}$, Facon $\mathrm{T}$, Goldschmidt $\mathrm{H}$, Jakubowiak A, et al. Improvement in Overall Survival With Carfilzomib, Lenalidomide, and Dexamethasone in Patients With Relapsed or Refractory Multiple Myeloma. J Clin Oncol. 2018;36(8):728-34.

13. Kaufman JL, Usmani SZ, San-Miguel J, Bahlis N, White DJ, Benboubker L, et al. Four-Year Follow-up of the Phase 3 Pollux Study of Daratumumab Plus Lenalidomide and Dexamethasone (DRd) Versus Lenalidomide and Dexamethasone (Rd) Alone in Relapsed or Refractory Multiple Myeloma (RRMM). Blood. 2019;134(Supplement_1):1866-.

14. Bringhen S, Petrucci MT, Larocca A, Conticello C, Rossi D, Magarotto V, et al. Carfilzomib, cyclophosphamide, and dexamethasone in patients with newly diagnosed multiple myeloma: a multicenter, phase 2 study. Blood. 2014;124(1):63-9.

15. Kaufman JL, Mina R, Jakubowiak AJ, Zimmerman TL, Wolf JJ, Lewis C, et al. Combining carfilzomib and panobinostat to treat relapsed/refractory multiple myeloma: results of a Multiple Myeloma Research Consortium Phase I Study. Blood Cancer J. 2019;9(1):3.

16. Costa LJ, Stadtmauer EA, Morgan G, 
Monohan G, Kovacsovics T, Burwick $N$, et al. Phase 2 Study of Venetoclax Plus Carfilzomib and Dexamethasone in Patients with Relapsed/Refractory Multiple Myeloma. Blood. 2018;132(Supplement 1):303-.

17. Moreau P, Harrison S, Cavo M, De La Rubia J, Popat R, Gasparetto C, et al. Updated Analysis of Bellini, a Phase 3 Study of Venetoclax or Placebo in Combination with Bortezomib and Dexamethasone in Patients with Relapsed/Refractory Multiple Myeloma. Blood. 2019;134 (Supplement_1):1888-.

18. Richardson PG, Schlossman RL, Alsina M, Weber DM, Coutre SE, Gasparetto C, et al. PANORAMA 2: panobinostat in combination with bortezomib and dexamethasone in patients with relapsed and bortezomib-refractory myeloma. Blood. 2013;122(14):2331-7.

19. Manasanch EE, Shah JJ, Lee HC, Weber DM, Thomas SK, Amini B, et al. Phase I/Ib study of carfilzomib and panobinostat with or without dexamethasone in patients with relapsed/refractory multiple myeloma. Haematologica. 2020;105(5):e242-e5.

20. Hoylman E, Brown A, Perissinotti AJ, Marini BL, Pianko M, Ye JC, et al. Optimal sequence of daratumumab and elotuzumab in relapsed and refractory multiple myeloma. Leuk Lymphoma. 2020;61(3):691-8.

21. Casneuf T, Xu XS, Adams HC, Axel AE, Chiu C, Khan I, et al. Effects of daratumumab on natural killer cells and impact on clinical outcomes in relapsed or refractory multiple myeloma. Blood Adv. 2017;1(23):2105-14.

22. Gerrie AS, Mikhael JR, Cheng L, Jiang H, Kukreti V, Panzarella T, et al. $\mathrm{D}(\mathrm{T}) \mathrm{PACE}$ as salvage therapy for aggressive or refractory multiple myeloma. Br J Haematol. 2013;161 (6):802-10.

23. Tabchi S, Nair R, Kunacheewa C, Patel $\mathrm{KK}$, Lee HC, Thomas SK, et al. Retrospective Review of the Use of High-Dose Cyclophosphamide, Bortezomib, Doxorubicin, and Dexamethasone for the Treatment of Multiple Myeloma and Plasma Cell Leukemia. Clin Lymphoma Myeloma Leuk. 2019;19(9):560-9.

24. Park S, Lee SJ, Jung CW, Jang JH, Kim SJ, Kim WS, et al. DCEP for relapsed or refractory multiple myeloma after therapy with novel agents. Ann Hematol. 2014;93(1):99-105.

25. Siegel DS, Martin T, Wang M, Vij R, Jakubowiak AJ, Lonial S, et al. A phase 2 study of single-agent carfilzomib (PX-171-003-A1) in patients with relapsed and refractory multiple myelo- ma. Blood. 2012;120(14):2817-25.

26. Lonial S, Weiss BM, Usmani SZ, Singhal S, Chari A, Bahlis NJ, et al. Daratumumab monotherapy in patients with treatment-refractory multiple myeloma (SIRIUS): an open-label, randomised, phase 2 trial. Lancet. 2016;387(10027):1551-60.

27. Chari A, Vogl DT, Gavriatopoulou M, Nooka AK, Yee AJ, Huff CA, et al. Oral Selinexor-Dexamethasone for TripleClass Refractory Multiple Myeloma. N Engl J Med. 2019;381(8):727-38.

28. Lonial S, Lee HC, Badros A, Trudel S, Nooka AK, Chari A, et al. Pivotal DREAMM-2 study: Single-agent belantamab mafodotin (GSK2857916) in patients with relapsed/refractory multiple myeloma (RRMM) refractory to proteasome inhibitors (PIs), immunomodulatory agents, and refractory and/or intolerant to anti-CD38 monoclonal antibodies (mAbs). Journal of Clinical Oncology. 2020;38 (15_suppl):8536-.

29. Mikhael J, Noonan KR, Faiman B, Gleason C, Nooka AK, Costa LJ, et al. Consensus Recommendations for the Clinical Management of Patients With Multiple Myeloma Treated With Selinexor. Clin Lymphoma Myeloma Leuk. 2020;20(6):351-7. 